\title{
Impact ionization and large room-temperature magnetoresistance in micron-sized high-mobility InAs channels
}

\author{
A. V. Velichko, ${ }^{1, *}$ O. Makarovsky, ${ }^{1}$ N. Mori, ${ }^{2}$ L. Eaves, ${ }^{1}$ A. Krier, ${ }^{3}$ Q. Zhuang, ${ }^{3}$ and A. Patanè ${ }^{1, \dagger}$ \\ ${ }^{1}$ School of Physics and Astronomy, the University of Nottingham, Nottingham NG7 2RD, UK \\ ${ }^{2}$ Graduate School of Engineering, Osaka University, 2-1 Yamada-Oka, Suita City, Osaka 565-0871, Japan \\ ${ }^{3}$ Physics Department, Lancaster University, Lancaster LA1 4YB, UK
}

(Received 14 May 2014; revised manuscript received 25 July 2014; published 20 August 2014)

\begin{abstract}
We report on hot electron induced impact ionization and large room-temperature magnetoresistance (MR) in micron-sized channels of $n$-type high-mobility $\operatorname{InAs}\left(\mu=3.3 \mathrm{~m}^{2} \mathrm{~V}^{-1} \mathrm{~s}^{-1}\right.$ at $\left.T=300 \mathrm{~K}\right)$ : the MR reaches values of up to $450 \%$ in magnetic fields of $1 \mathrm{~T}$ and applied voltages of $\sim 1 \mathrm{~V}$ and is weakly dependent on temperature. We present Monte Carlo simulations of the hot electron dynamics to account for the large MR and its dependence on the sample geometry and applied electric and magnetic fields. Our work demonstrates that the impact ionization of electrons at room temperature, under small applied magnetic fields $(<1 \mathrm{~T})$ and small voltages $(<1 \mathrm{~V})$, can provide an extremely sensitive mechanism for controlling the electrical resistance of high-mobility semiconductors.
\end{abstract}

DOI: 10.1103/PhysRevB.90.085309

PACS number(s): 72.20.-i, 78.55.Cr, 78.40.Fy

\section{INTRODUCTION}

Elucidating the mechanisms of avalanche breakdown due to impact ionization in semiconductors is crucial to our understanding of the physics of nonlinear transport phenomena in these materials and harnessing them for device applications. Among these are avalanche photodiodes (APDs) [1], magnetoresistive sensors (e.g., magnetic recording devices, magnetic memory, position and speed sensors) [2] and reconfigurable semiconductor logic components [3]. On the one hand, avalanche breakdown limits the high-power performance of transistors [4] and semiconductor junctions [5] due to the large increase of current induced by the rapid multiplication of carriers at high electric fields. On the other hand, impact ionization is exploited in APDs to reach the high gain (10-30) and low excess noise factor $(\leq 2)[4,6,7]$ required for sensing in the near- to midinfrared spectral range $(\lambda=1-5 \mu \mathrm{m})$ in applications such as atmospheric gas detection and radiation thermometry [8].

In a strong applied electric field, E, carriers can gain enough kinetic energy to ionize dopant impurities at low temperature [9-11] and/or to generate electron-hole pairs by interband impact ionization leading to avalanche breakdown $[5,12,13]$, which greatly increases the conductivity. A magnetic field, B, applied perpendicular to the direction of the current flow, can strongly affect these impact ionization processes by increasing the binding energy of electrons bound onto impurities and hence the activation energy and electric field required for impurity ionization [9-11]; also, the Lorentz force exerted on the conduction electrons deflects the electron motion away from the direction of the electric field, effectively increasing the electric field required for excitation of electrons across the band gap. Thus a large magnetoresistance (MR) can result from the effect of a magnetic field on the hot carrier dynamics $[11,14,15]$, although this often requires low temperatures

\footnotetext{
*Corresponding author: anton.velychko@nottingham.ac.uk

†Corresponding author: amalia.patane@nottingham.ac.uk
}

and/or applied voltages in excess of tens or even hundreds of volts.

In this paper we report on the MR of short-channel (2$5 \mu \mathrm{m})$ planar devices based on $n$-type InAs epilayers with high electron mobility $\left(\mu=3.3 \mathrm{~m}^{2} \mathrm{~V}^{-1} \mathrm{~s}^{-1}\right.$ at $\left.T=300 \mathrm{~K}\right)$ and narrow band gap $\left(\varepsilon_{g}=0.35 \mathrm{eV}\right.$ at $\left.T=300 \mathrm{~K}\right)$. We show that the electronic properties of InAs are well suited for achieving a large room-temperature transverse MR (of strength up to $450 \%)$ at low applied magnetic fields $(\leq 1 \mathrm{~T})$ and modest applied voltages $(V \sim 1 \mathrm{~V})$. Our Monte Carlo simulations of the electron dynamics demonstrate that the large MR is caused by impact ionization and that the sample geometry can be designed to enhance this MR phenomenon. These findings are of general interest as they apply to other high-mobility, narrow band gap semiconductors. Also, they are of technological relevance, as magnetic-field dependent impact ionization can provide a highly sensitive mechanism for controlling the electrical resistance without the need to exploit spin-related transport phenomena, as is commonly done in magnetic materials [16-18].

\section{EXPERIMENTAL}

The nominally undoped high-mobility InAs epilayer (thickness $t=1.5 \mu \mathrm{m}$ ) was grown by molecular beam epitaxy (MBE) on a semi-insulating (SI) (100)-oriented GaAs substrate. The GaAs substrate provides effective isolation for device fabrication. Magnetotransport studies of Hall bars fabricated from the InAs epilayer were used to determine an electron Hall mobility of $\mu=3.3 \mathrm{~m}^{2} \mathrm{~V}^{-1} \mathrm{~s}^{-1}\left(3.1 \mathrm{~m}^{2} \mathrm{~V}^{-1} \mathrm{~s}^{-1}\right)$ at $T=$ $300 \mathrm{~K}(4.2 \mathrm{~K})$ and an electron density $n_{e}=4 \times 10^{16} \mathrm{~cm}^{-3}$ at $T=300 \mathrm{~K}$, which is weakly dependent on $T$. Due to the high conductivity of InAs $(\sigma>2 \mathrm{~S} / \mathrm{cm})$, the Hall coefficient in the Hall bar-shaped samples cannot be measured at large applied electric fields (i.e., in the regime of impact ionization) as this would produce currents in excess of $I=10$ A. Thus, here we focus on short and narrow channel devices. The InAs epilayer was processed into two-terminal planar devices with InAs channels of length, $L$, and width, $W$, in the range of 


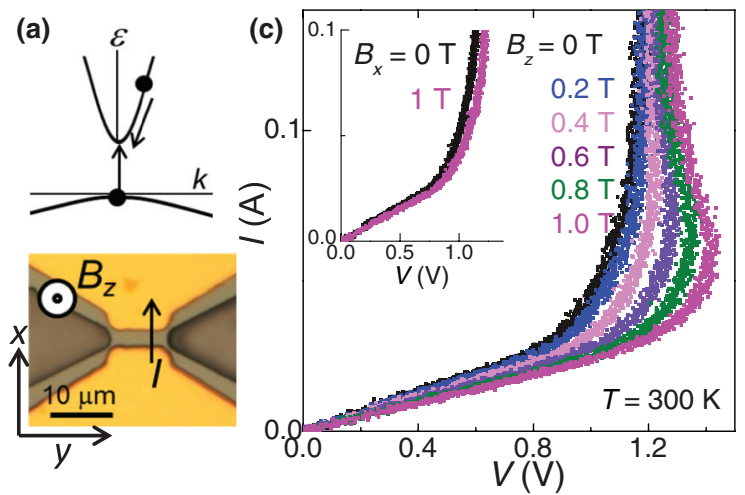

(b)
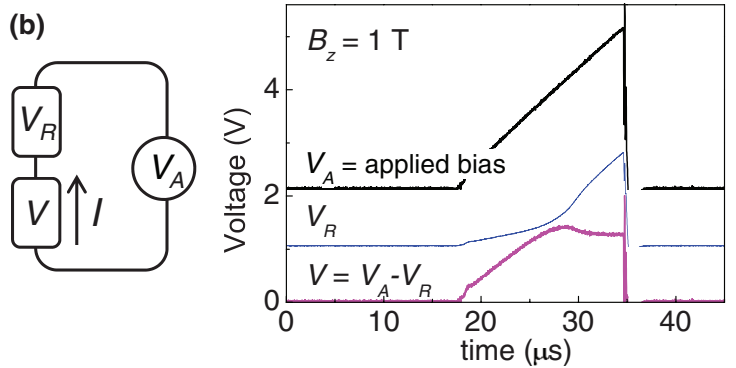

FIG. 1. (Color online) (a) Sketch of the conduction band (CB) and valence band (VB) of InAs showing the excitation of an electron from the VB to the $\mathrm{CB}$ caused by impact ionization. The bottom inset shows the optical image of a two-terminal InAs planar device (length $L=2.6 \mu \mathrm{m}$ and width $W=10 \mu \mathrm{m}$ ) and the direction of the magnetic field, $B_{z}$, and current, $I$. (b) Measuring circuit and time dependence of the applied bias $\left(V_{A}\right)$ and voltages, $V_{R}$ and $V$, dropped across the series resistor and device, respectively $\left(T=300 \mathrm{~K}\right.$ and $\left.B_{z}=1 \mathrm{~T}\right)$. (c) Dependence of the current, $I$, on voltage, $V$, at various $B_{z}$ for the device shown in panel a $(T=300 \mathrm{~K})$. The inset shows the $I$ - $V$ curves at magnetic fields $B_{x}$ applied parallel to the current $(T=300 \mathrm{~K})$.

2-5 $\mu \mathrm{m}$ and 5-10 $\mu \mathrm{m}$, respectively [Fig. 1(a)]. Each channel was formed by dry-etching the epilayer down to the GaAs substrate. Metal contacts consisting of $10 \mathrm{~nm}$ of Ti followed by $200 \mathrm{~nm}$ of $\mathrm{Au}$ were deposited onto the samples to form Ti-Au Ohmic contacts.

An earlier paper demonstrated that Hall bars and twoterminal devices based on InAs exhibit a linear MR over an extended range of applied magnetic fields $\mathbf{B}$ up to $50 \mathrm{~T}$ [19]. Here we focus on the effects of impact ionization on the MR at low applied magnetic fields up to $1 \mathrm{~T}$. The magnetic field B was generated either by a superconducting magnet or a room-temperature electromagnet and was applied parallel or perpendicular to the growth axis $z$; i.e., $\mathbf{B}=\left[0,0, B_{z}\right]$ or $\mathbf{B}=\left[B_{x}, 0,0\right]$. In all experiments $\mathbf{E}$ was applied along $x$; $i$.e., $\mathbf{E}=\left[E_{x}, 0,0\right]$.

Measurements of the current-voltage $I-V$ characteristics were performed in the pulsed electric field regime using a high-speed (100 MS/s real-time sampling), high-resolution (12-bit) scope coder and a custom-made ramp generator that delivers short $(\sim 10 \mu \mathrm{s})$ single "sawtooth" voltage pulses of amplitude $V_{A}$ that increase in time from 0 up to a maximum value. This voltage is applied to a circuit comprising the device and a series resistor $(R=10 \Omega)$. The voltage, $V$, across the device is derived from the applied voltage $V_{A}$ by subtracting the measured voltage $V_{R}$ across the series resistor; i.e., $V=V_{A}-V_{R}$; the current, $I$, is then given by $I=V_{R} / R$ [see Fig. 1(b)]. This technique allowed us to apply large electric fields (up to tens of kilovolts per centimeter) and pass high pulsed currents (up to several hundred milliamperes) without causing thermal breakdown and degradation of the devices, which would be unavoidable in dc measurements. Also, the use of short pulses avoids temporal instabilities of the current, which can occur at high electric fields due to filamentary current flow [10].

\section{RESULTS}

We first consider the perpendicular field configuration, $\mathbf{B}=\left[0,0, B_{z}\right]$. Figure $1(\mathrm{c})$ shows the room temperature $I-V$ characteristics for a device with $L=2.6 \mu \mathrm{m}$ and $W=10 \mu \mathrm{m}$ and $B_{z}$ values from 0 to $1 \mathrm{~T}$. At each $B_{z}$, the $I-V$ curves are approximately linear at low bias. The Ohmic behavior is then followed by a sublinear bias dependence and, at larger biases, by a sharp rise of the current and an S-shaped $I-V$ dependence, which is characteristic of systems switching from a state of low to high conductivity due to an increase in the impact ionization rate [13]. Figure 1(b) shows the time dependence of the applied bias, $V_{A}$, and the voltages, $V_{R}$ and $V$, across the resistor and device, respectively. It can be seen that as $V_{A}$ increases with time, $V_{R}$ and $V$ also increase. However, as the current and hence $V_{R}$ start increasing more rapidly (due to impact ionization), the voltage $V$ across the device decreases. Thus at a given $V$, we can measure two values of the current and probe the S-shaped $I-V$ characteristic. We note that this dependence differs from the quadratic dependence of $I$ on $V$ due to space-charge-induced MR and bipolar-charge-injection previously reported for Si-based devices [15].

At $B_{z}=0 \mathrm{~T}$ the threshold bias for the rapid rise of the current is $V_{t h}=0.94 \mathrm{~V}$, which corresponds to a threshold electric field $E_{t h}=V_{t h} / L=3.6 \mathrm{kV} / \mathrm{cm}$, where $L=2.6 \mu \mathrm{m}$. This value is in agreement with previous studies of $n$-type InAs epilayers, which reported an increase in impact ionization rate and conductivity at similar electric fields [20]. Increasing $B_{z}$

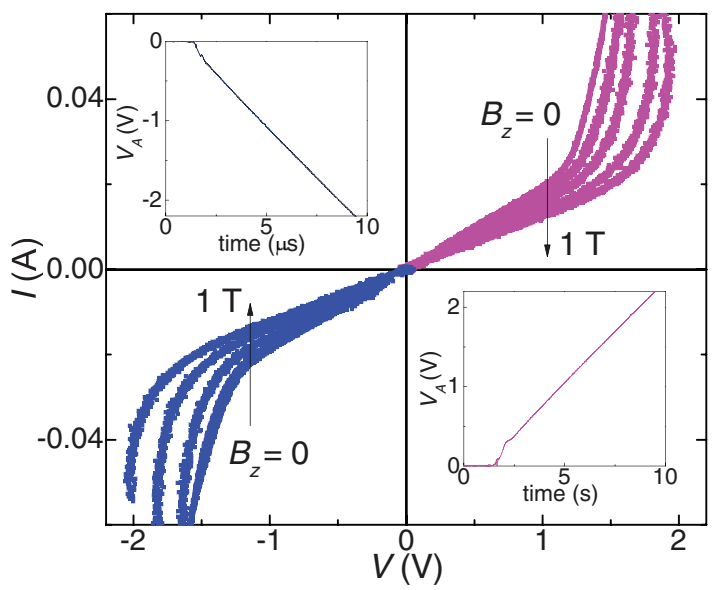

FIG. 2. (Color online) Dependence of the current, $I$, on voltage, $V$, at various $B_{z}=0,0.25,0.5,0.75$, and $1 \mathrm{~T}$ for an InAs planar device of length $L=5 \mu \mathrm{m}$ and width $W=10 \mu \mathrm{m}(T=300 \mathrm{~K})$. The insets show the time dependence of the short applied bias $\left(V_{A}\right)$ pulse used to acquire the $I-V$ curve for $V>0$ and $V<0$. 

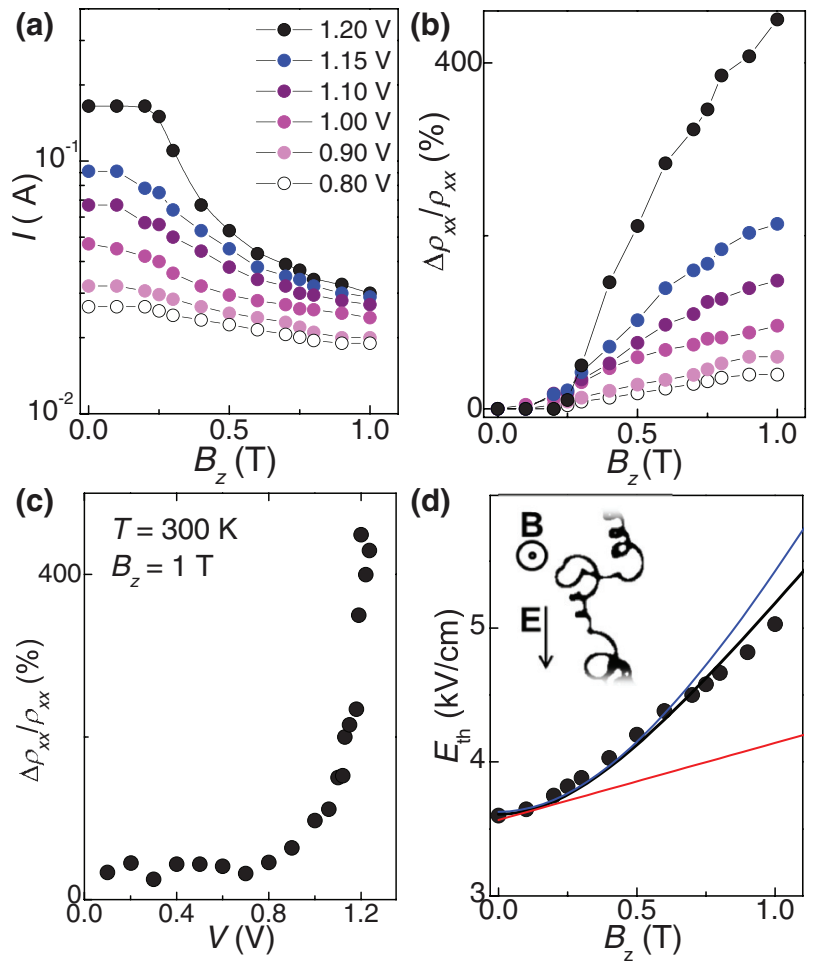

FIG. 3. (Color online) (a) Current, $I$, as a function of magnetic field, $B_{z}$, at various voltages, $V(T=300 \mathrm{~K})$. Lines are guides to the eye. (b) Transverse MR, $\Delta \rho_{x x} / \rho_{x x}$, as a function of $B_{z}$ at various $V$ at $T=300 \mathrm{~K}$. (c) Transverse MR, $\Delta \rho_{x x} / \rho_{x x}$, as a function of $V$ at $T=300 \mathrm{~K}$ and $B_{z}=1 \mathrm{~T}$. (d) Dependence of the electric field for impact ionization, $E_{t h}$, on $B_{z}$ at $T=300 \mathrm{~K}$. Lines are the calculated values of $E_{t h}$ according to the model described in the text using a two-band model (black line), parabolic (blue line), and linear (red curve) energy dispersions. All data are for an InAs channel of length $L=2.6 \mu \mathrm{m}$ and width $W=10 \mu \mathrm{m}$. The inset sketches the electron cyclotron motion with magnetic field, $\mathbf{B}$, perpendicular to the electric field, E.

has the effect of increasing $V_{t h}$ up to about $1.3 \mathrm{~V}$ at $B_{z}=1 \mathrm{~T}$, effectively stretching out the $I-V$ curve to higher voltages. We obtained similar $I-V$ curves for other devices with different lengths and/or widths of the InAs conducting channel; the $I-V \mathrm{~s}$ are reproducible in time and are symmetric with respect to positive and negative applied biases (see Fig. 2). Also, we note that the $I-V$ curves change very little when the magnetic field is applied along $x$, parallel to the direction of $\mathbf{E}$ [see inset of Fig. 1(c)]. Thus, in what follows, we will concentrate only on the perpendicular magnetic field geometry; i.e., $\mathbf{B}=$ $\left[0,0, B_{z}\right]$.

As shown in Fig. 3(a), for each applied bias $V$, the current decreases with increasing $B_{z}$. The $B_{z}$-induced suppression of the current becomes stronger at high applied voltages and results in a transverse MR ratio, $\Delta \rho_{x x} / \rho_{x x}=\left[\rho_{x x}\left(B_{z}\right)-\right.$ $\left.\rho_{x x}(0)\right] / \rho_{x x}(0)$, that is strongly dependent on $V$, reaching a value of up to $450 \%$ at $B_{z}=1 \mathrm{~T}$ and $V \sim 1 \mathrm{~V}$ [see Figs. 3(b) and 3(c)]. In our derivation of $\Delta \rho_{x x} / \rho_{x x}$ for $V$ in the range of the S-shaped $I-V$ curve, we have used the lowest value of the current. With reference to Fig. 1(b), this value of the current is measured at the time when impact ionization is first achieved at each voltage pulse.
The measured MR values are comparable to those reported in the literature for MR devices based on magnetic materials [16-18] and, more recently, for nonmagnetic semiconductors; e.g., Si [15] and InSb [3]. Large values of the MR $\left(\sim 10^{3} \%\right.$ at $T=300 \mathrm{~K}$ and $B \sim 1 \mathrm{~T}$ ) were also reported in hybrid metal semiconductor devices [21,22]. However, our observations are qualitatively different from these previous studies as, in our case, the large MR is caused by the effect of the applied magnetic field in suppressing impact ionization of high-mobility electrons in our micrometer InAs conducting channels. The following uses a semiclassical model and Monte Carlo simulations of the electron dynamics to examine this phenomenon.

In the $\mathbf{B}=\left[0,0, B_{z}\right]$ configuration, the Lorentz force tends to increase the electron momentum component along $y$, perpendicular to $\mathbf{B}=\left[0,0, B_{z}\right]$ and $\mathbf{E}=\left[E_{x}, 0,0\right]$. The corresponding loss of kinetic energy for electron motion along $x$ implies that, with increasing $B_{z}$, a larger electric field is required to sustain the electron conduction along $x$, thus shifting the impact ionization threshold to higher electric field values. Because of the high electron mobility $(\mu=$ $3.3 \mathrm{~m}^{2} \mathrm{~V}^{-1} \mathrm{~s}^{-1}$ at $T=300 \mathrm{~K}$ ), magnetic fields as small as $B_{z}=0.2 \mathrm{~T}$ can induce an increase of the electric field for impact ionization $E_{t h}$, and hence a corresponding increase of the MR [Figs. 3(c) and 3(d)]. Also, because of the narrow band gap of InAs $\left(\varepsilon_{g}=0.35 \mathrm{eV}\right.$ at $\left.T=300 \mathrm{~K}\right)$ and short channel length $(L=2.6 \mu \mathrm{m})$, small applied voltages $(V \sim 1 \mathrm{~V})$ are sufficient to initiate the electron impact ionization.

In stark contrast to many MR phenomena observed in several other material systems in which the strength of the MR tends to quench with increasing temperature [14,23-25], in our short InAs channels the large MR is observed over an extended temperature range. This is illustrated in Fig. 4(a) where the $I-V$ curves are plotted for various $B_{z}$ at $T=2,100$, and $300 \mathrm{~K}$. With increasing temperature, the voltage required for impact ionization tends to decrease [Fig. 4(a)], but the dependence of the MR on the applied bias is similar at all $T$ [Fig. 4(b)]. The dependence of $E_{t h}$ on temperature is influenced by the temperature dependence of the fundamental band gap energy, $\varepsilon_{g}$, and carrier mobility, $\mu$. Since an increasing $T$ from 2 to $300 \mathrm{~K}$ tends to reduce the band gap of InAs from $\varepsilon_{g}=0.42$ to $0.35 \mathrm{eV}$, the threshold electric field for interband impact ionization should decrease; the effect of temperature on the electron mobility, $\mu$, should also be taken into account, and this can vary in different material systems and structures. For our $n$-type InAs layer, the change with temperature of $\mu$ is small: an increase of $T$ from 2 to $300 \mathrm{~K}$ leads to an increase of $\mu$ from 3.1 to $3.3 \mathrm{~m}^{2} / \mathrm{Vs}$ at $300 \mathrm{~K}$ due to weaker impurity scattering. This small increase $(\Delta \mu / \mu=6 \%)$ of electron mobility with increasing temperature should also decrease the value of $E_{t h}$, although this is a small effect compared to the stronger decrease $(\Delta \varepsilon / \varepsilon=-17 \%)$ of the band gap energy.

\section{DISCUSSION}

\section{A. Semiclassical model}

For an insight into the magnetic field dependence of the threshold electric field for impact ionization, $E_{t h}$, we first consider a simple semiclassical model. For $\mathbf{B}=\left[0,0, B_{z}\right]$ 


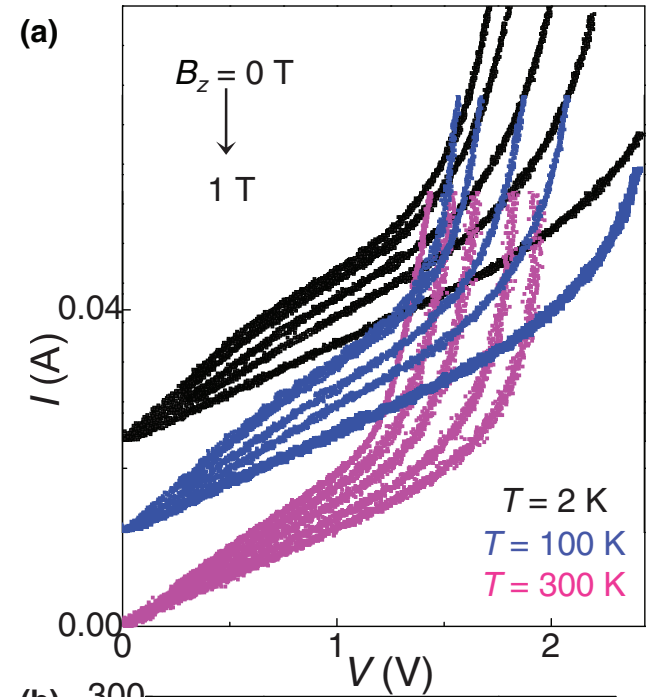

(b)

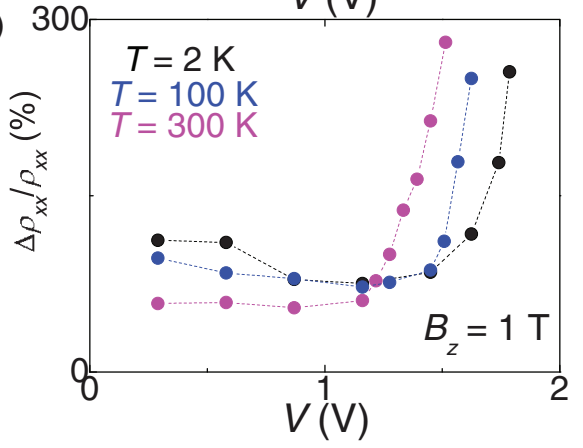

FIG. 4. (Color online) (a) Dependence of the current, $I$, on voltage, $V$, at various magnetic fields, $B_{z}$, and temperatures, $T$. The magnetic field increases from 0 to 1 in steps of $0.2 \mathrm{~T}(T=2 \mathrm{~K}$ and $100 \mathrm{~K})$ or $0.25 \mathrm{~T}(T=300 \mathrm{~K})$. For clarity, curves at different $T$ are shifted along the vertical axis. (b) Transverse MR, $\Delta \rho_{x x} / \rho_{x x}$, as a function of $V$ at $B_{z}=1 \mathrm{~T}$ and various $T$. All data are for an InAs channel of length $L=5.8 \mu \mathrm{m}$ and width $W=10 \mu \mathrm{m}$.

and $\mathbf{E}=\left[E_{x}, 0,0\right]$, the Lorentz force increases the electron momentum component along $y$, perpendicular to $\mathbf{E}=\left[E_{x}, 0\right.$, 0 ], according to $\hbar k_{y}=e B_{z} s$, where $s$ is the distance travelled by the electrons along the $x$ direction. The corresponding loss of kinetic energy for electron motion along $x$ implies that a larger electric field $E_{x}=E_{t h}$ is required to sustain the electron conduction along $x$ and hence to impact ionize electrons moving from the source to the drain electrode. The energy loss depends on the values of $B_{z}$ and $s$ and on the form of the energy dispersion, $\varepsilon\left(k_{y}\right)$. We use a nonparabolic conduction band dispersion to describe the kinetic energy along $y$; i.e., $\varepsilon\left(k_{y}\right)\left[1+\alpha \varepsilon\left(k_{y}\right)\right]=\hbar^{2} k_{y}^{2} / 2 m_{e}^{*}$, where $\alpha=2.2 \mathrm{eV}^{-1}$ and $m_{e}^{*}=0.026 m_{e}$ is the electron effective mass at $k=0$ [26]. Hence we approximate the energy required for impact ionization along $x$ as $\varepsilon_{t h}\left(B_{z}\right)=\varepsilon_{t h}(0)+\varepsilon\left(k_{y}\right)$, where $\varepsilon_{t h}(0)$ is the impact ionization threshold at $B_{z}=0 \mathrm{~T}$, which we set equal to $\varepsilon_{t h}(0)=1.1 \varepsilon_{g}$ [27] with $\varepsilon_{\mathrm{g}}=0.35 \mathrm{eV}$ at $T=$ $300 \mathrm{~K}$. The increase of $\varepsilon_{t h}\left(B_{z}\right)$ with increasing $B_{z}$ corresponds to an increase of the electron wave vector, $k_{t h}$, for motion along $x$ and of the threshold electric field $E_{x}=E_{t h}=\hbar k_{t h} / e \tau$, where $\tau$ is the electron scattering time that is,

$$
E_{t h}\left(B_{z}\right)=\frac{\hbar k_{t h}\left(B_{z}\right)}{e \tau}=\frac{1}{e \tau} \sqrt{2 m_{e}^{*} \varepsilon_{t h}\left(B_{z}\right)\left[1+\alpha \varepsilon_{t h}\left(B_{z}\right)\right]},
$$

where

$$
\varepsilon_{t h}\left(B_{z}\right)=\varepsilon_{t h}(0)+\frac{1}{2 \alpha}\left[\sqrt{1+\frac{2 \alpha e^{2} B_{z}^{2} s^{2}}{m_{e}^{*}}}-1\right] .
$$

In Fig. 3(d), the measured dependence of $E_{t h}$ on $B_{z}$ is well described by Eqs. (1) and (2) with $\tau=1.9 \times 10^{-12}$ $\mathrm{s}$ and $s=0.30 \mu \mathrm{m}$ (black line). Simplified expressions of the energy dispersion (i.e., parabolic $\varepsilon=\hbar^{2} k^{2} / 2 m_{e}^{*}$ and linear $\left.\varepsilon=\hbar k / \sqrt{2 \alpha m_{e}^{*}}\right)$, give simpler expressions for $E_{t h}\left(B_{z}\right)$, but a poorer description of the data. The parabolic energy dispersion gives a stronger magnetic field dependence given by $E_{t h}(B)=E_{t h}(0) \sqrt{1+\omega_{c}^{2} \tau_{1}^{2}}$, where $\omega_{c}=e B_{z} / m_{e}^{*}$ is the cyclotron frequency and $\tau_{1}=s \sqrt{m_{e}^{*} / 2 \varepsilon_{t h}(0)}=0.17 \times 10^{-12} \mathrm{~s}$, corresponding to $s=0.38 \mu \mathrm{m}$ [see blue curve in Fig. 3(d)]. The linear "relativistic" energy dispersion gives instead a weaker $B_{z}$ dependence given by $E_{t h}(B)=E_{t h}(0)\left(1+\omega_{c} \tau_{2}\right)$, where $\omega_{c}=e B_{z} /\left[2 m_{e}^{*} \alpha \varepsilon_{t h}(0)\right]$ and $\tau_{2}=s \sqrt{2 \alpha m_{e}^{*}}=0.04 \times 10^{-12} \mathrm{~s}$, corresponding to $s=0.05 \mu \mathrm{m}$ [see red curve in Fig. 3(d)].

\section{B. Monte Carlo simulations}

Equations (1) and (2) are simple analytical expressions that provide us with an empirical explanation of the change in $E_{t h}$ as the magnetic field increases. However, these equations are based on several simplifying assumptions, including the assumption that $E_{y}=0$, and the separability of the electron motion along $x$ and $y$. We examine further the effect of an applied magnetic field on the ionization of carriers by Monte Carlo simulations of the hot electron dynamics for two different geometries referred to as G1 and G2. In the G1 geometry, the Hall field is "shorted-out" (i.e., $E_{y}=0$ ) and the $y$ component of the current density is nonzero (i.e., $J_{y} \neq 0$ ); in the $\mathrm{G} 2$ geometry, we assume $J_{y}=0$ and $E_{y} \neq 0$. As the magnetic field is applied along the $z$ direction, the magnetic field affects the components of the electron motion in the $x y$ plane. Thus in our simulation of the electron dynamics, we treat the InAs layer as a two-dimensional (2D) conductor. Also, we assume that the impact ionization is initiated by electrons, which are much lighter than heavy holes in InAs; e.g., $m_{h h}^{*} / m_{e}^{*}$ $>10$. The nonparabolicity of the electron energy dispersion of InAs is modeled as $\varepsilon(\mathbf{k})[1+\alpha \varepsilon(\mathbf{k})]=\hbar^{2} k^{2} / 2 m_{e}$, where $m_{e}=$ $0.0265 m_{o}$ and $\alpha=2.2 \mathrm{eV}^{-1}$. The electron velocity, given by $\boldsymbol{v}(\boldsymbol{k})=\hbar^{-1} \nabla \varepsilon$, and the wave vector $\boldsymbol{k}$ change with time under the action of the Lorentz force $\mathbf{F}=-e[\mathbf{E}+\boldsymbol{v} \times \mathbf{B}]$.

We next consider the effect of disorder-induced scattering on the electron dynamics. Since our InAs epilayers are grown on highly lattice-mismatched GaAs, threading dislocations tend to form at the epilayer/substrate interface and introduce macroscopic $(>0.1 \mu \mathrm{m})$ inhomogeneities, leading to strong linear MR, as reported in Ref. [19]. In this previous paper, inhomogeneity was introduced in the Monte Carlo simulations by placing low-mobility islands randomly within the $x y$ plane. The random spatial profile of the islands $\Delta(x, y)$ is 
TABLE I. Value of the inelastic $\left(r_{i}\right)$ and elastic $\left(r_{e}\right)$ scattering rates, the correlation length $(\Lambda)$, and coverage factor $(f)$ of lowmobility islands used in the Monte Carlo simulations of the electron dynamics at $T=300 \mathrm{~K}$.

\begin{tabular}{lccc}
\hline \hline$r_{i}$ & $r_{e}$ & $\Lambda$ & $f$ \\
\hline $1.5 \times 10^{12} \mathrm{~s}^{-1}$ & $2.5 \times 10^{14} \mathrm{~s}^{-1}$ & $0.5 \mu \mathrm{m}$ & 0.05 \\
\hline \hline
\end{tabular}

generated from the power spectrum of the autocorrelation function $\left\langle\Delta(\mathbf{r}) \Delta\left(\mathbf{r}^{\prime}\right)\right\rangle=\Delta^{2} \exp \left[-\left|\mathbf{r}-\mathbf{r}^{\prime}\right|^{2} / \Lambda^{2}\right]$, where $\Lambda$ is the correlation length, $\mathbf{r}=(x, y)$, and $\mathbf{r}^{\prime}=\left(x^{\prime}, y^{\prime}\right)$. The lowmobility regions $R_{\text {low }}$ are then defined according to the relation $R_{\text {low }}=\left\{\mathbf{r} \mid \Delta(\mathbf{r})<\Delta_{\text {th }}\right\}$. This condition determines the fractional area, $f$, of the sample with low mobility in which electrons undergo scattering at a rate higher than in the other parts of the sample. In the low-mobility regions, we use a scattering rate $r_{\text {island }}=r_{i}+r_{e}$, where $r_{e}$ and $r_{i}$ are the elastic and inelastic phonon scattering rates, respectively; elsewhere, the electron drift is mainly limited by inelastic scattering with $r \sim r_{i}$. This model describes well our observation of linear MR at low applied electric fields and high $B_{z}$ with $r_{i}=1.5 \times 10^{12}$ $\mathrm{s}^{-1}, r_{e}=2.5 \times 10^{14} \mathrm{~s}^{-1}, \Lambda=0.5 \mu \mathrm{m}$ and $f=0.05$ (Table I; Ref. [19]).

We focus now on the effects of impact ionization on the MR at large applied electric fields. To account for the effect of the impact ionization on the MR, we monitor the electron kinetic energy $\varepsilon(t)$ for a sufficiently long simulation time interval $T_{\text {sim }}$ and count the number of occurrences of impact ionization processes $N_{i i}$. Here we assume that an impact ionization process occurs when $\varepsilon(t)$ exceeds the threshold energy $\varepsilon_{t h}$ and that the electron thermalizes after the impact ionization. Hence we calculate the electron impact ionization rate $R_{i i}$ $\left(=N_{i i} / T_{\text {sim }}\right)$ versus $E_{x}$ and $B_{z}$ and the corresponding impact ionization coefficient $\alpha_{i i}=R_{i i} / v$, where $v$ is the electron speed.

At $B_{z}=0$, electron impact ionization occurs when $\alpha_{i i} L_{t h}=$ 1 , where $L_{t h}$ is a characteristic impact ionization length. By setting $\alpha_{i i}$ to the value corresponding to $E_{x}$ equal to the measured value of $E_{t h}$ at $B_{z}=0\left(E_{t h}=3.6 \mathrm{kV} / \mathrm{cm}\right)$, we find that $L_{t h}=\alpha_{i i}^{-1}=2.2 \mu \mathrm{m}$ at $B_{z}=0$. Thus, while traveling from the source at $x=0$ to the drain at $x=L=2.6 \mu \mathrm{m}$, an electron experiences impact ionization very close to the drain. For $B_{z}$ $>0$, electrons travel at a Hall angle $\theta$ to the direction of $E_{x}$, so the distance along $x$ that an electron requires to travel from the source to drain before an impact ionization event occurs is $L_{t h} / \cos \theta$. Thus the condition for impact ionization becomes $R_{i i} L_{t h} / v \cos \theta=\alpha_{i i_{x}} L_{t h}=1$, where $\alpha_{i i_{x}}=R_{i i} / v_{x}$ and $v_{x}$ is the component of the electron velocity along $x$. We use this condition to determine $E_{t h}$ at $B_{z}>0$. As shown in Figs. 5(a) and 5(b), the calculated value of $\alpha_{i i_{x}}$ increases with increasing $E_{x}$ at all $B_{z}$ and for both geometries G1 [Fig. 5(a)] and G2 [Fig. 5(b)], although a stronger $B_{z}$ dependence is obtained in the G1 geometry.

Figure 5(c) shows the calculated dependence of $E_{t h}$ on $B_{z}$ for geometries G1 $\left(E_{y}=0\right.$; red curve) and G2 $\left(J_{y}=\right.$ 0 ; blue curve). Both geometries indicate an increase of $E_{t h}$ with increasing $B_{z}$, with a stronger magnetic field dependence observed for $E_{y}=0$. For $E_{y}=0$, the calculated $E_{t h}\left(B_{z}\right)$

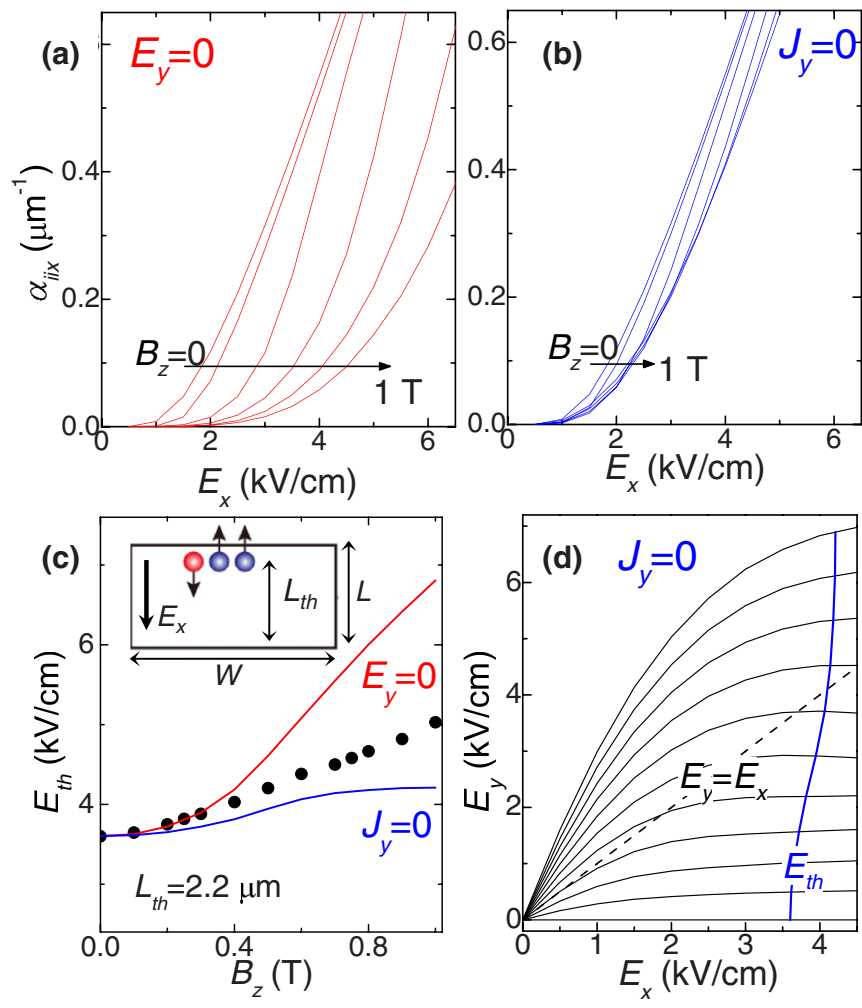

FIG. 5. (Color online) (a, b) Impact ionization coefficient, $\alpha_{i i_{x}}$, as a function of $E_{x}$ at $B_{z}=0,0.2,0.4,0.6,0.8$, and $1.0 \mathrm{~T}$ for geometries $\mathrm{G} 1\left(E_{y}=0\right)$ and $\mathrm{G} 2\left(J_{y}=0\right)$. (c) Calculated threshold electric field for impact ionization, $E_{t h}$, versus $B_{z}$ for geometry $\mathrm{G} 1$ (red curve) and G2 (blue curve) for an impact ionization length of $L_{t h}$ $=2.2 \mu \mathrm{m}$. Symbols are the experimental data. The inset sketches the impact ionization of electrons along a channel of length $L$ and width $W$. (d) Dependence of $E_{y}$ on $E_{x}$ at various $B_{z}$ in the G2 geometry $\left(J_{y}=0\right)$. The dashed line corresponds to the condition $E_{y}=E_{x}$. The blued line shows the dependence of $E_{t h}$ on $B_{z}$.

curve deviates from the experimental data (symbols) when $B_{z}$ $>0.3 \mathrm{~T}$, which corresponds to a Hall angle $\theta>20^{\circ}$. For $B_{z}$ $>0.3 \mathrm{~T}$, the measured data are closer to those calculated for $J_{y}=0$ [blue curve in Fig. 5(c)]. Also, for $J_{y}=0$, the data reveal an inflection point at $B_{z} \approx 0.5-0.6 \mathrm{~T}$, which is also predicted by the model. As shown in Fig. 5(d), the inflection point in the $E_{t h}$ versus $B_{z}$ curve occurs at a value of $B_{z}$ at which the Hall field $E_{y}$ becomes equal to $E_{x}$.

\section{Edge effects}

Since neither of the geometries G1 and G2 reproduce quantitatively the measured dependence $E_{t h}\left(B_{z}\right)$, we deduce that neither G1 nor G2 describe the real geometry due to sample edge effects; i.e., the equipotential and current flow lines are nonuniform in a real device. To assess how these effects influence the impact ionization, we solve numerically the Laplace equation $\nabla^{2} \phi(x, y)=0$ for a device of length $L=$ $2.6 \mu \mathrm{m}$ and width $W=10 \mu \mathrm{m}$, using the boundary conditions described in Refs. [28-30] and indicated in Fig. 6. As shown in Fig. 6 close to the sample edges (at $y \sim 0$ and $y \sim 10 \mu \mathrm{m}$ ), 


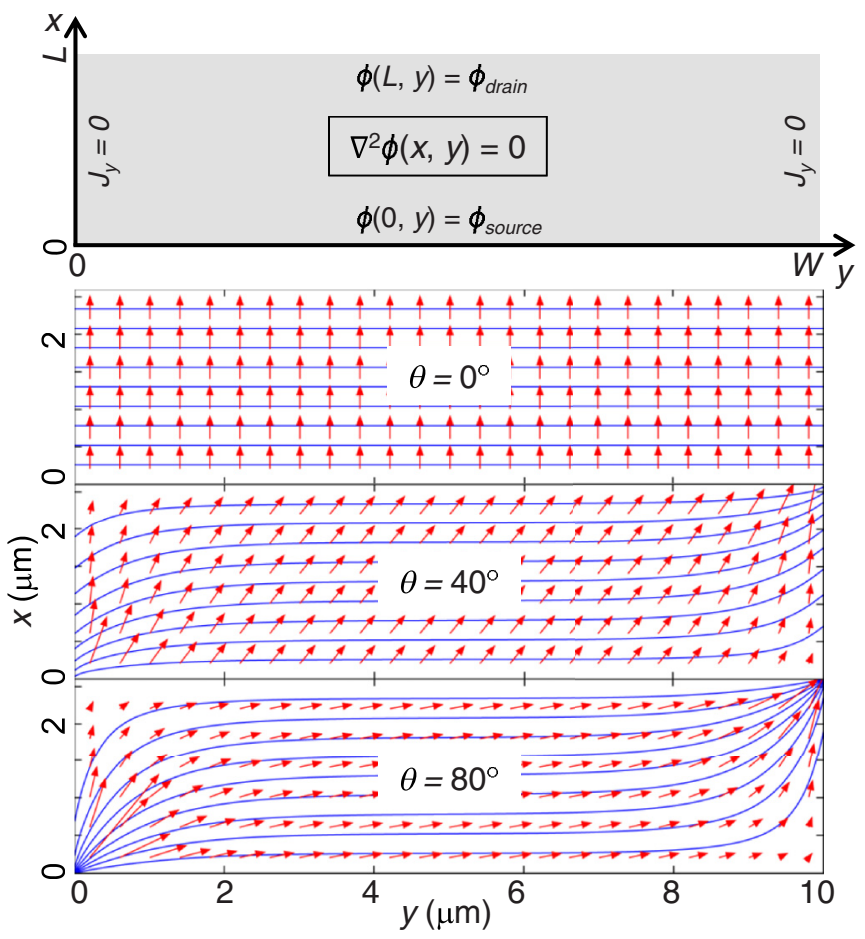

FIG. 6. (Color online) At the top is a sketch of a conducting channel of length $L$ and width $W$, and of the boundary conditions used to solve numerically the Laplace equation $\nabla^{2} \phi(x, y)=0$ and to calculate the equipotential lines (blue lines) and current flow vector field (red vectors) shown in the bottom for a Hall angle $\theta$ equal to $\theta=0^{\circ}, \theta=40^{\circ}$ and $\theta=80^{\circ}$.

the calculated current flows along the edges, regardless of the value of $B_{z}$ or Hall angle $\theta$. In contrast, the current is tilted at an angle $\theta$ in the center of the channel $(y \sim 5 \mu \mathrm{m})$. For electrons traveling near the edges $(\theta=0), v_{y} \sim 0$. In these regions the dependence of $E_{t h}$ on $B_{z}$ should be weak, as indicated by the Monte Carlo simulations for the G2 geometry $\left(J_{y}=0\right)$ [see blue curve in Fig. 5(c)]. On the contrary, away from the edges, electrons travel at an angle $\theta$, and the dependence of $E_{t h}$ on $B_{z}$ should be stronger, as indicated by the Monte Carlo simulations for the G1 geometry [see red curve in Fig. 5(c)]. Thus we conclude that in our sample the avalanche carrier multiplication occurs primarily near the edges of the device and that the measured values of $E_{t h}$ should lie between those calculated for the two different geometries G1 and G2.

Although edge effects were not included in the Monte Carlo simulations, our simplified discussions in the two different geometries G1 and G2 provide us with at least a qualitative understanding of the effect of the Hall field on the strength of the MR and indicate that larger MR values could be obtained for geometry G1 (i.e., for short channels $[L \ll W]$ ) and Corbino geometries [21], in which the Hall electric field is fully "shorted out" by the contact electrodes. For our samples, we have chosen a geometry in which $L$ is smaller than $W$. Figure 7 shows the room temperature $I-V$ characteristics for three devices with $L=2.6,2.4$, and $5.8 \mu \mathrm{m}$ and $W=10,5$, and $10 \mu \mathrm{m}$ at $B_{z}=0$ and $1 \mathrm{~T}(T=300 \mathrm{~K})$ corresponding to an aspect ratio $L / W$ of $0.26,0.48$ and 0.58 . In all structures we have measured a large room-temperature MR, exceeding

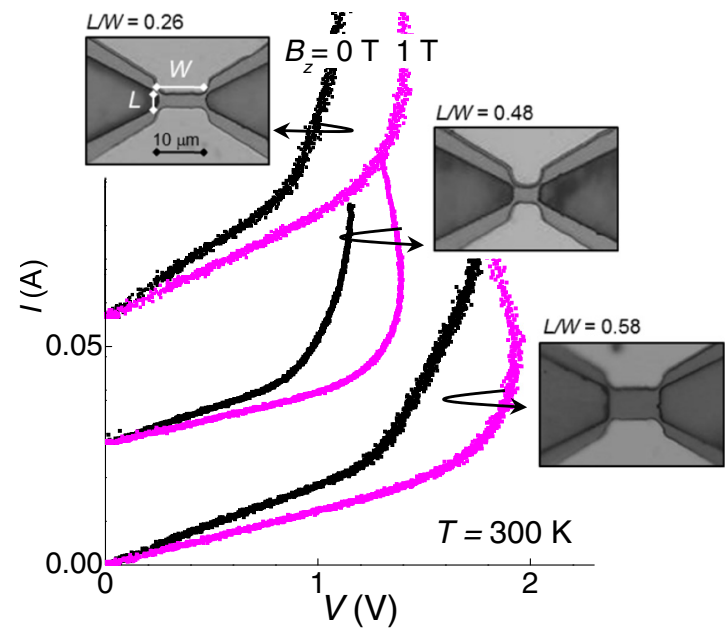

FIG. 7. (Color online) Dependence of the current, $I$, on voltage, $V$, at $B_{z}=0$ and $1 \mathrm{~T}(T=300 \mathrm{~K})$ for InAs channels with different values of the aspect ratio $L / W$, where $L$ and $W$ are the length and width of the channel, respectively. The insets show optical images of the devices. For clarity, curves for different devices are shifted along the vertical axis.

$100 \%$ at $B_{z}=1 \mathrm{~T}$ and $V \sim 1 \mathrm{~V}$; also, we note that the presence of nonhomogeneities in the layers affects the strength of the MR [19], thus making it difficult to identify a systematic dependence on the aspect ratio $L / W$.

\section{CONCLUSION}

In summary, we have observed room-temperature MR of up to $\sim 450 \%$ in high-mobility InAs conducting channels ( $\mu=$ $3.3 \mathrm{~m}^{2} \mathrm{~V}^{-1} \mathrm{~s}^{-1}$ ) at applied magnetic fields of $1 \mathrm{~T}$ and voltages $V \sim 1 \mathrm{~V}$. The threshold field for impact ionization $E_{t h}$ is strongly enhanced by a perpendicular magnetic field (from 0 to $1 \mathrm{~T}$ ), resulting in a large MR that is only weakly affected by temperature. Our observations are described by Monte Carlo simulations of the hot electron dynamics; also, our data and analysis show that the sample geometry should be carefully designed to optimize the dependence of the impact ionization threshold field on the applied magnetic field, with a larger MR expected in Corbino-like geometries or short-channel devices where the Hall field is shorted out. These findings demonstrate that impact ionization can provide a highly sensitive mechanism for controlling the electrical resistance of high-mobility, narrow band gap semiconductors such as InAs. This class of materials can provide an attractive alternative to magnetic materials for achieving large MR effects at room temperature and low applied biases.

\section{ACKNOWLEDGMENTS}

This work was supported by the Engineering and Physical Sciences Research Council (EPSRC) (EP/J015296/1). We are grateful to Dr. R. Airey (EPSRC, III-V National Facility, Sheffield) for processing our samples. 
[1] I. C. Sandall, J. S. Ng, S. Xie, P. J. Ker, and C. H. Tan, Opt. Express 21, 8630 (2013).

[2] J. Heremans, J. Phys. D Appl. Phys. 26, 1149 (1993).

[3] S. Joo, T. Kim, S. H. Shin, J. Y. Lim, J. Hong, J. D. Song, J. Chang, H. W. Lee, K. Rhie, S. H. Han, K. H. Shin, and M. Johnson, Nature 494, 72 (2013).

[4] B. Kochman, K. Yeom, and J. Singh, Appl. Phys. Lett. 68, 1936 (1996).

[5] J. Allam and A. R. Adams, Phys. Status Solidi B 211, 335 (1999).

[6] A. Marshall, J. P. R. David, and C. H. Tan, IEEE Trans. Electron. Dev. 57, 2631 (2010).

[7] S. J. Maddox, W. Sun, Z. Lu, H. P. Nair, J. C. Campbell, and S. R. Bank, Appl. Phys. Lett. 101, 151124 (2012).

[8] Y. Shimizu, J. Ishii, Y. Kaneko, F. Sakuma, and A. Ono, SICE 2004 Annual Conference, Sapporo, Japan (IEEE, Piscataway, NJ, 2004), Vol. 2, pp. 1803-1806.

[9] V. A. Samuilov, V. K. Ksenevich, G. Remenyi, G. Kiss, and B. Pödör, Semicond. Sci. Technol. 14, 1084 (1999).

[10] F. S. Lee and Y. C. Cheng, Phys. Rev. B 56, 6412 (1997).

[11] J. J. H. M. Schoonus, P. P. J. Haazen, H. J. M. Swagten, and B. Koopmans, J. Phys. D: Appl. Phys. 42, 185011 (2009).

[12] G. Satyanadh, P. R. Joshi, N. Abedin, and U. Singh, J. Appl. Phys. 91, 1331 (2002).

[13] O. Makarovsky, W. H. M. Feu, A. Patanè, L. Eaves, Q. D. Zhuang, A. Krier, R. Beanland, and R. Airey, Appl. Phys. Lett. 96, 052115 (2010).

[14] M. P. Delmo, S. Yamamoto, S. Kasai, T. Ono, and K. Kobayashi, Nature 457, 1112 (2009)
[15] M. P. Delmo, E. Shikoh, T. Shinjo, and M. Shiraishi, Phys. Rev. B 87, 245301 (2013).

[16] H. Akinaga, M. Mizuguchi, K. Ono, and M. Oshima, Appl. Phys. Lett. 76, 357 (2000).

[17] K. H. Gao, Q. W. Wang, T. Lin, Z. Q. Li, X. H. Zhang, J. Xu, H. Y. Deng, and J. H. Chu, Europhys. Lett. 102, 37009 (2013).

[18] M. Yokoyama, T. Ogawa, A. M. Nazmul, and M. Tanaka, J. Appl. Phys 99, 08D502 (2006).

[19] N. V. Kozlova, N. Mori, O. Makarovsky, L. Eaves, Q. D. Zhuang, A. Krier, and A. Patanè, Nature Commun. 3, 1097 (2012).

[20] M. V. Fischetti, IEEE Trans. Electron Devices 38, 634 (1991).

[21] S. A. Solin, T. Thio, D. R. Hines, and J. J. Heremans, Science 289, 1530 (2000).

[22] J. Sun and J. Kosel, Materials 6, 500 (2013).

[23] J. J. H. M. Schoonus, F. L. Bloom, W. Wagemans, H. J. M. Swagten, and B. Koopmans, Phys. Rev. Lett. 100, 127202 (2008).

[24] T. Yu and P. Chen, IEEE Trans. Magnetics 47, 3467 (2011),

[25] N. Overend, A. Nogaret, B. L. Gallagher, P. C. Main, M. Henini, C. H. Marrows, M. A. Howson, and S. P. Beaumont, Appl. Phys. Lett. 72, 1724 (1998).

[26] C. Hamaguchi, Basic Semiconductor Physics (Springer-Verlag, London, 2006), p. 46.

[27] P. T. Landsberg and Y. J. Yu, J. Appl. Phys. 63, 1789 (1988).

[28] R. F. Wick, J. Appl. Phys. 25, 741 (1954).

[29] G. De Mey, Adv. Electron. Electron Phys. 61, 1 (1983).

[30] T. Mimizuka, Solid-State Electron. 14, 107 (1971). 